Nervenarzt 2021 · 92:144-149 https://doi.org/10.1007/s00115-020-00999-6 Angenommen: 3. August 2020 Online publiziert: 1 . Oktober 2020 (c) Der/die Autor(en) 2020

Felix Kleefeld' für Deutsche Gesellschaft für Neuro-AIDS und Neuro-Infektiologie (DGNANI) - Gabriele Arendt ${ }^{2}$ für Deutsche Gesellschaft für Neuro-AIDS und Neuro-Infektiologie (DGNANI) - Eva Neuen-Jacob ${ }^{3}$ für Deutsche Gesellschaft für Neuro-AIDS und Neuro-Infektiologie (DGNANI) · Matthias Maschke für Deutsche Gesellschaft für Neuro-AIDS und Neuro-Infektiologie (DGNANI) - Ingo Husstedt ${ }^{5}$ für Deutsche Gesellschaft für Neuro-AIDS und Neuro-Infektiologie (DGNANI) Mark Obermann ${ }^{6}$ für Deutsche Gesellschaft für Neuro-AIDS und NeuroInfektiologie (DGNANI) • Holger Schmidt ${ }^{7}$ für Deutsche Gesellschaft für NeuroAIDS und Neuro-Infektiologie (DGNANI) · Katrin Hahn ${ }^{1}$ für Deutsche Gesellschaft für Neuro-AIDS und Neuro-Infektiologie (DGNANI)

${ }^{1}$ Klinik für Neurologie, Universitätsmedizin Charité, Berlin, Deutschland

${ }^{2}$ Neurologie, Neuro-Centrum Düsseldorf, Düsseldorf, Deutschland

${ }^{3}$ Institut für Neuropathologie, Universitätsklinikum Düsseldorf, Düsseldorf, Deutschland

${ }^{4}$ Klinik für Neurologie, Krankenhaus der Barmherzigen Brüder, Trier, Deutschland

${ }^{5}$ Praxis an der Klinik Maria Frieden, Telgte/Münster, Deutschland

${ }^{6}$ Klinik für Neurologie, Asklepios Kliniken Schildautal, Seesen, Deutschland

${ }^{7}$ Klinik für Neurologie, Elbe-Kliniken Stade, Stade, Deutschland

\title{
Neurologische Komplikationen der Hepatitis-C-Infektion
}

\section{Hintergrund}

Geschätzt 180 Mio. Menschen sind weltweit mit dem Hepatitis-C-Virus (HCV) infiziert. In Deutschland lassen sich bei etwa $0,5 \%$ der Bevölkerung Antikörper gegen HCV nachweisen [1]. Die Hepatitis $\mathrm{C}$ ist somit eine hochprävalente und im klinischen Alltag häufig anzutreffende Infektionskrankheit. Seit 2014 lässt sich die chronische HCV-Infektion durch den Einsatz direkt wirkender antiviraler Substanzen (,direct acting antivirals", DAAs) effektiv und nebenwirkungsarm behandeln und bei $>90 \%$ der Patienten dauerhaft heilen [2]. Die heute verfügbaren DAAs umfassen Proteaseinhibitoren, NS5A- sowie NS5B-Inhibitoren und greifen somit gezielt in den Replikationszyklus des Virus ein.

Es ist gut belegt, dass HCV nicht nur hepatotrop, sondern auch lymphotrop wirksam ist $[3,4]$. Dies bedingt, dass das Virus neben einer chronisch-aktiven Hepatitis auch eine Vielzahl extrahepatischer Manifestationen verursachen kann. Einige Autoren verwenden daher den Begriff „Hepatitis-C-Syndrom“ [5], um das Spektrum extrahepatischer spezifischer Organmanifestationen, systemischer Autoimmunerkrankungen und Tumoren im Kontext der Infektion zu charakterisieren. Extrahepatische Manifestationen sind häufig Ausdruck einer B-Zell-Proliferation mit Produktion mono- oder polyklonaler Antikörper. Damit assoziiert ist beispielsweise das Auftreten einer Kryoglobulinämie [6, 7]. Kryoglobuline lassen sich entsprechend der Brouet-Klassifikation [8] in 3 Typen einteilen; wobei sich der II. und III. Typ, welche auch als gemischte Kryoglobulinämie bezeichnet werden, häufig bei Hepatitis-C-Infektionen nachweisen lassen $[9,10]$. Das Spektrum der Antikörper umfasst darüber hinaus sowohl antinukleäre Antikörper, Anti SS-A/Anti-SS-B, ANCA als auch GM1-Gangliosid-Antikörper [11]. Der Phänotyp des HCVSyndroms scheint multifaktoriell bedingt zu sein, einschließlich genetischer und geografischer Faktoren, sodass sich regionale Unterschiede in der Manifestation ergeben können [6]. Aus diesem Grund ist die Erkrankung klinisch nicht nur für Internisten relevant. Für Neurologen und Psychiater von Bedeutung sind die Manifestationen im zentralen und peripheren Nervensystem sowie der Muskulatur. Die HCV-Infektion wird darüber hinaus mittlerweile als eigenständiger Risikofaktor für verschiedene neurologische (z.B. Demenz, Morbus Parkinson), kardiovaskuläre (z. B. Atherosklerose, Schlaganfall) und metabolische (Diabetes mellitus) Erkrankungen angesehen [12]. In dieser Übersichtsarbeit geben wir einen Überblick über mögliche neurologische Komplikationen der HCV-Infektion (• Abb. 1).

\section{Peripheres Nervensystem und Muskulatur}

Das periphere Nervensystem (PNS) ist bei HCV-infizierten Patienten insbesondere durch Polyneuropathien betroffen. Das Spektrum möglicher Erkrankungen beinhaltet distal-symmetrische, häufig schmerzhafte axonale Polyneuropathien (PNP), Small-fiber-Neuropathien (SFN) und seltener auch akute oder chronische Polyradikuloneuropathien wie das Guillain-Barré-Syndrom (GBS) oder die chronische inflammatorische demyelinisierende Polyradikuloneuropathie (CIDP). Auch asymmetrische Neuropa- 


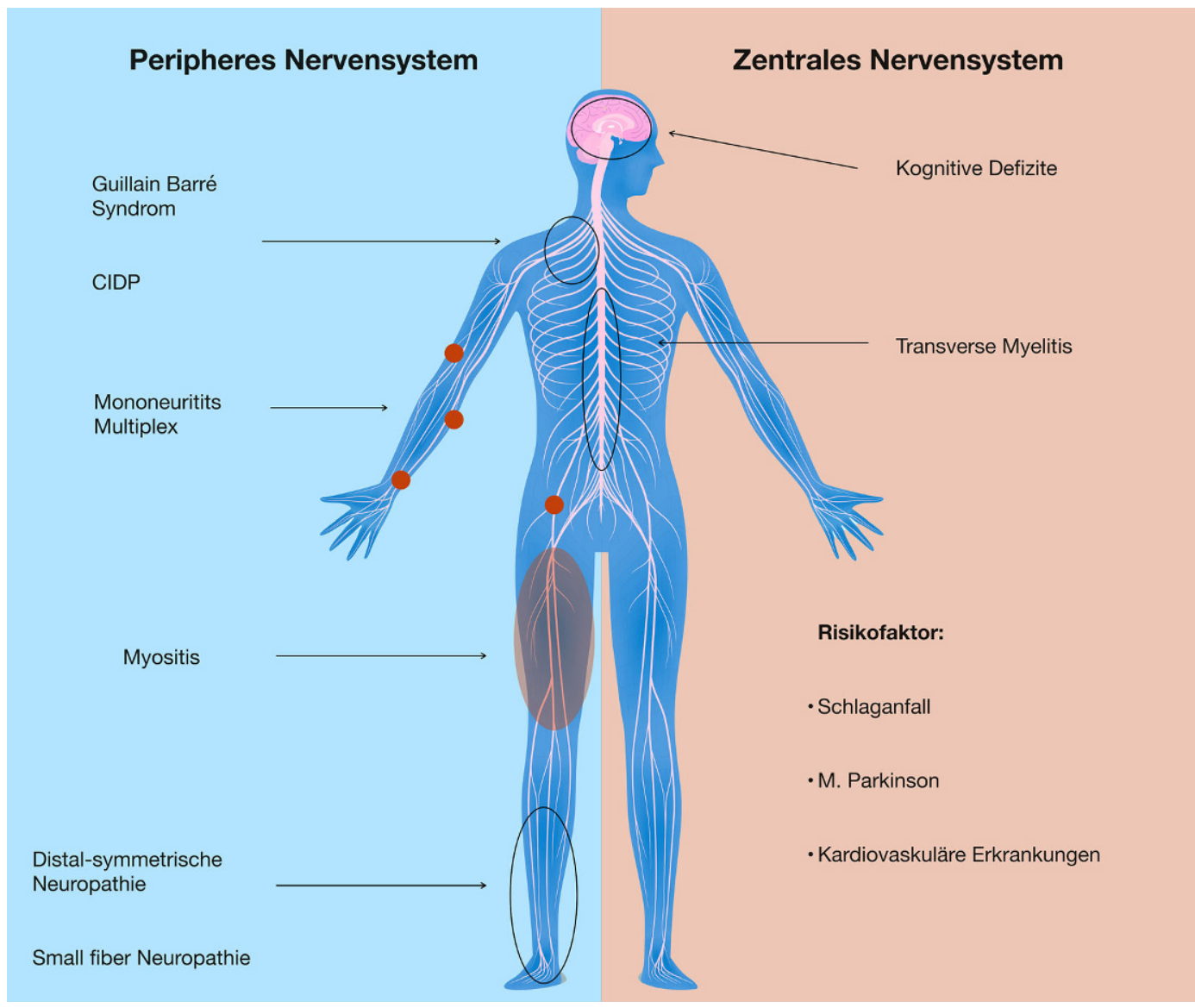

Abb. $1<$ Überblick der wichtigsten neurologischen Komplikationen der Hepatitis-C-Infektion. CIDP chronische inflammatorische demyelinisierende Polyneuropathie

thien wie die Mononeuritis multiplex sind beschrieben. Es lassen sich damit chronische (PNP, SFN) von akutsubakut auftretenden (CIDP, GBS) und axonale von demyelinisierenden Neuropathien im Kontext der HCV-Infektion unterscheiden.

Angaben zur Prävalenz Hepatitis-Cassoziierter Neuropathien finden sich in der Literatur kaum. Eine prospektive französische Arbeit mit 321 Patienten beschreibt in $9 \%$ das Vorliegen einer sensiblen Polyneuropathie [13]. Mehrheitlich präsentieren sich Patienten mit einer symmetrischen sensiblen oder sensomotorischen Polyneuropathie und deutlich seltener mit einer Mononeuropathie oder Mononeuropathia multiplex [14, 15].

Die Pathogenese der HCV-assoziierten Neuropathien ist nur unvollständig verstanden. Neben vaskulitischen Veränderungen der Vasa nervosum mit Komplementaktivierung und sekundärer Ischämie werden auch direkte virusabhängige Mechanismen mit sekundärer
Inflammation diskutiert [15-17]. HCVRNA ließ sich in epineuralen Zellen nachweisen $[17,18]$.

Unabhängig vom Auftreten von Kryoglobulinen finden sich in Suralisbiopsien HCV-positiver Patienten mit PNP gehäuft perivaskuläre inflammatorische Infiltrate mit mononukleären Zellen [19], was die Immunpathogenese der Neuropathien unterstreicht. Neben der Präsenz von Kryoglobulinen vom Typ II und III (gemischte Kryoglobulinämie) ist insbesondere die Erkrankungsdauer ein Risikofaktor für das Auftreten von PNP bei diesen Patienten [10, 20]. Der HCVGenotyp, Schweregrad der Leberfibrose sowie der Kryokrit, also der prozentuale Anteil präzipitierter Kryoglobuline, gelten hingegen nicht als Risikofaktoren für das Auftreten einer PNP [20].

\section{Distal-symmetrische Polyneu- ropathien und Small-fiber- Neuropathien}

Distal-symmetrische, sensomotorische axonale PNP stellen eine der häufigsten Komplikationen der HCV-Infektion dar und sind mit dem Auftreten von Kryoglobulinen (CG) und einer Vaskulitis assoziiert. Bis zu $86 \%$ der kryoglobulinpositiven Patienten, aber auch bis $\mathrm{zu}$ $44 \%$ der kryoglobulinnegativen Patienten sind von einer PNP betroffen [21]. Somit ist das Vorliegen einer HCV-assoziierten Polyneuropathie auch ohne Nachweis von Kryoglobulinen möglich. Klinisch zeigt sich bei den betroffenen Patienten eine häufig schmerzhafte, vorwiegend sensible und distal-symmetrische Polyneuropathie, die in der Regel einen schleichenden bzw. chronischen Verlauf aufweist. Es bestehen Hinweise, dass bei kryoglobulinnegativen Patienten häufiger auch asymmetrische Neuropathien vom Multiplex-Typ auftreten [10]. Eine Hirnnervenbeteiligung ist bei al- 
len Formen von $\mathrm{HCV}$-assoziierter PNP beschrieben [10].

Neben klassischen Polyneuropathien leiden HCV-positive Patienten auch unter teils isoliert auftretenden Smallfiber-Neuropathien. Im Gegensatz zur klassischen (sog. Large-fiber-)PNP bestehen bei reinen SFN keine Atrophien, Paresen und keine Areflexie. Patienten mit SFN können sich mit isolierten, schmerzhaften Parästhesien, Temperaturempfindungsstörungen oder autonomen Funktionsstörungen präsentieren. Diese initial oft schwer zuzuordnenden Symptome werden erfahrungsgemäß häufig als Schmerzsyndrome, Fibromyalgie oder Somatisierungsstörung fehldiagnostiziert. Die klinisch-neurologische Untersuchung erbringt ebenso wie die Neurographie bei diesen Patienten in der Regel unauffällige Befunde. Die Diagnose SFN lässt sich mit verschiedenen Methoden diagnostizieren. Je nach Verfügbarkeit kann z.B. eine histomorphologische Beurteilung der intraepidermale Nervenfaserdichte in einer Hautstanzbiopsie erfolgen oder eine funktionelle Charakterisierung z. B. mittels quantitativer sensorischer Testung. Bei Diagnose einer SFN sollten daher unbedingt auch Infektionserkrankungen wie die HCV- oder die häufig auch komorbide vorliegende HIV-Infektion in die differenzialdiagnostischen Überlegungen mit einbezogen werden.

\section{Therapie}

Bei der Therapie der HCV-assoziierten Polyneuropathien ist zwischen der symptomorientierten Basistherapie, im Falle einer kryoglobulinassoziierten vaskulitischen Neuropathie einer immunsuppressiven und einer Hepatitis-C-spezifischen antiviralen, kausalen Therapie der Polyneuropathie $\mathrm{zu}$ unterscheiden.

Neuropathische Schmerzen können u. a. mit Antikonvulsiva, Antidepressiva, Opiaten oder topischen Therapien (z. B. Capsaicin-Pflaster) nach herkömmlichen symptomatischen Prinzipien behandelt werden. Aus unserer Erfahrung hat sich bei diesen Patienten aber z.B. Duloxetin gut wirksam gezeigt. Kontrollierte Studien fehlen. In einer offenen Studie mit Patienten mit schmerzhaf-

Nervenarzt 2021 · 92:144-149 https://doi.org/10.1007/s00115-020-00999-6

(c) Der/die Autor(en) 2020

F. Kleefeld für Deutsche Gesellschaft für Neuro-AIDS und Neuro-Infektiologie (DGNANI) . G. Arendt für Deutsche Gesellschaft für Neuro-AIDS und Neuro-Infektiologie (DGNANI) E. Neuen-Jacob für Deutsche Gesellschaft für Neuro-AIDS und Neuro-Infektiologie (DGNANI) • M. Maschke für Deutsche Gesellschaft für Neuro-AIDS und Neuro-Infektiologie (DGNANI) . I. Husstedt für Deutsche Gesellschaft für Neuro-AIDS und Neuro-Infektiologie (DGNANI) . M. Obermann für Deutsche Gesellschaft für Neuro-AIDS und Neuro-Infektiologie (DGNANI) . H. Schmidt für Deutsche Gesellschaft für Neuro-AIDS und Neuro-Infektiologie (DGNANI) K. Hahn für Deutsche Gesellschaft für Neuro-AIDS und Neuro-Infektiologie (DGNANI)

\section{Neurologische Komplikationen der Hepatitis-C-Infektion}

\section{Zusammenfassung}

Die chronische Hepatitis-C-Virus(HCV)Infektion ist eine hochprävalente Systemerkrankung, die verschiedene neurologische Komplikationen verursachen kann. Es lassen sich HCV-assoziierte Symptome im zentralen und peripheren Nervensystem sowie der Muskulatur unterscheiden. Wichtige Pathomechanismen sind die HCVassoziierte Autoimmunität (z. B. gemischte Kryoglobulinämie mit Polyneuropathie) und direkte Neurotoxizität (z. B. bei HCVassoziierten kognitiven Defiziten). Die häufigsten neurologischen Komplikationen sind distal-symmetrische Polyneuropathien, Small-fiber-Neuropathien und kognitive
Defizite. Die HCV-Infektion stellt außerdem einen Risikofaktor für ischämische und hämorrhagische Schlaganfälle sowie den Morbus Parkinson dar. Die frühe Identifikation und antivirale Behandlung HCV-positiver Patienten steht im Zentrum der Behandlung. Durch neue antivirale Therapien können $>90 \%$ der Patienten dauerhaft von der HCVInfektion geheilt werden.

Schlüsselwörter

Hepatitis C · Polyneuropathie · Kognitive Defizite $\cdot$ Kryoglobulinämie $\cdot$ Small-fiberNeuropathie

\section{Neurological complications of hepatitis C infections}

\section{Abstract}

Chronic hepatitis C virus (HCV) infection is a highly prevalent systemic disease, which can cause a variety of neurological complications. The HCV-associated symptoms can be differentiated into central and peripheral nervous systems as well as the musculature. Important pathomechanisms are HCV-associated autoimmunity (e.g. mixed cryoglobulinemia with polyneuropathy) and direct neurotoxic effects of the virus (e.g. $\mathrm{HCV}$-associated cognitive deficits). Distal symmetric polyneuropathies, small fiber neuropathies and cognitive deficits are the most prevalent neurological manifestations. Furthermore, $\mathrm{HCV}$ infection is a risk factor for ischemic and hemorrhagic stroke as well as Parkinson's disease. As HCV infection has become a permanently curable disease in $>90 \%$ of patients, early identification and antiviral treatment of HCV positive patients is of utmost importance.

\section{Keywords}

Hepatitis C · Polyneuropathy · Cognitive deficits · Cryoglobulinemia - Small fiber neuropathy ter Hepatitis-C-assoziierter kryoglobulinämischer Neuropathie zeigte sich ein positiver Effekt von Oxcarbazepin in der Schmerzkontrolle [22].

Bei Patienten mit kryoglobulinassoziierter vaskulitischer Neuropathie muss in Abhängigkeit von der Akuität der Erkrankung der additive Einsatz immunsuppressiver Substanzen wie Steroide, Cyclosporin bis hin zur Plasmapherese diskutiert werden [23, 24].

Grundsätzlich sollte aber bei allen Patienten eine kausale Therapie der
HCV-Infektion angestrebt werden. Die Auswahl des entsprechenden Therapieregimes sollte dabei in infektiologischer Hand liegen. Daten zur Wirksamkeit der neuen DAA-basierten Therapien auf Polyneuropathien liegen aktuell noch nicht vor. Da interferonbasierte Therapien aber in der Vergangenheit zum Teil deutliche Verbesserungen der Neuropathien erzielten, ist von einer mindestens vergleichbaren Wirksamkeit der DAAs auszugehen $[14,16]$. 
Demyelinisierende Polyneuropathien/Polyradikulopathien

Das GBS und die CIDP stellen akute bzw. subakut-chronisch auftretende, immunvermittelte Neuropathien dar. Sie wurden sowohl bei der akuten, als auch der chronischen HCV-Infektion beschrieben [25, 26].

Die immunmodulierende Akuttherapie des HCV-assoziierten GBS bzw. der CIDP unterscheidet sich nicht von der der nicht-HCV-assoziierten Varianten. Sie kann die Gabe von Immunglobulinen, Immunsuppressiva oder Plasmapherese beinhalten. Allerdings konnte in der Vergangenheit gezeigt werden, dass eine zusätzliche antivirale Therapie mit Interferon die Prognose möglicherweise zusätzlich verbessert [27, 28]. Die Wirksamkeit der DAAs in diesem Kontext ist bislang nicht untersucht worden.

\section{Myopathien}

Neben dem PNS kann auch die Muskulatur in seltenen Fällen Manifestationsort extrahepatischer Symptome der HCV-Infektion sein. Insbesondere das Auftreten der Polymyositis und der Einschlusskörpermyositis („inclusion body myositis“, IBM) ist mit der chronischen Hepatitis C assoziiert [29]. So waren bei $28 \%$ der Patienten mit IBM in einer Studie HCV-Antikörper nachweisbar [30]. Die Assoziation der IBM zu einer viralen Infektion ist insofern plausibel, als auch die HIV-Infektion einen etablierten Risikofaktor für das Auftreten einer IBM, insbesondere bei jüngeren Patienten, darstellt. In neueren Untersuchungen profitierten $\mathrm{HCV}$-positive Patienten mit IBM allerdings hinsichtlich ihrer neurologischen Symptomatik nicht von einer Viruseradikation, sodass eine therapeutische Konsequenz der HCV-Infektion im Kontext der IBM nicht gesichert ist [31]. - Die Differenzialdiagnose von distalsymmetrischen, schmerzhaften Neuropathien sowie Schwerpunktneuropathien sollte immer die Hepatitis-CInfektion beinhalten.

- Isolierte Small-fiber-Neuropathien können sich als klinisch blande, schmerzhafte Neuropathien präsentieren. Zur Diagnosestellung ist in unklaren Fällen eine Hautbiopsie notwendig.

- Immunvermittelte Neuropathien und Myopathien stellen seltene Komplikationen der HCV-Infektion dar.

- Neben symptomatischen Therapiemaßnahmen sollte bei allen Patienten mit HCV-assoziierter Neuropathie eine antivirale Therapie der Grunderkrankung angestrebt werden.

\section{Zentrales Nervensystem}

Die HCV-Infektion kann auch im zentralen Nervensystem (ZNS) verschiedene Komplikationen hervorrufen. Diese umfassen Enzephalitiden, Myelitiden, kognitive Defizite sowie Fatigue. Im Rahmen einer fortgeschrittenen Leberfibrose bei chronischer HCV-Infektion kann es außerdem zu einer hepatischen Enzephalopathie (HE) kommen. Diese Komplikation in Form einer metabolischen Enzephalopathie ist allerdings nicht HCVspezifisch und wird daher an dieser Stelle nicht besprochen. Pathophysiologisch sind im Gegensatz zur metabolischen Genese bei der HE andere Mechanismen für die ZNS-Komplikationen bei der HCVInfektion relevant. Zum einen wird eine direkte und indirekte Neurotoxizität des Hepatitis-C-Virus im ZNS angenommen. Die aktive Replikation des Virus im ZNS lässt sich auch durch die Virämie im Liquor nachweisen. Daneben spielen in der Pathogenese offensichtlich auch die Folgen einer chronischen Immunaktivierung eine Rolle, wie z.B. Autoimmunität und eine Dysfunktion der Blut-HirnSchranke durch Sekretion proinflammatorischer Zytokine wie Interleukin 1ß (IL$1 ß$; [32]).

\section{Kognitive Defizite}

Die Erstbeschreibung kognitiver Defizite bei HCV-infizierten Patienten ohne strukturelle Leberveränderungen im Jahre 2002 führte zu einer anhaltenden wissenschaftlichen Debatte [33]. Während viele Arbeiten die Existenz kognitiver Defizite bei HCV-infizierten Patienten reproduzieren konnten, stellten einige Autoren mit ihren Ergebnissen die klinische Relevanz der detektierten Defizite infrage [34]. Bei ausführlichen neuropsychologischen Untersuchungen HCVpositiver Patienten zeigten die Patienten allerdings in den meisten Studien reproduzierbare Defizite in bestimmten kognitiven Domänen. Es handelt sich dabei um die Bereiche Lernen und (Arbeits-)Gedächtnis, Aufmerksamkeit, Konzentration, Exekutivfunktionen und Feinmotorik [35]. Die HCV-Infektion scheint zu einem charakteristischen Muster kognitiver Defizite zu führen, das möglicherweise auf virusbedingte strukturelle Veränderungen und neuroinflammatorische Prozesse im ZNS zurückzuführen ist. Hier besteht klinisch und möglicherweise auch pathophysiologisch eine $\mathrm{Pa}$ rallele zum HIV-assoziierten kognitiven Defizit (HAND), das ein ähnliches neuropsychologisches Muster aufweist. Das HCV-Syndrom kann zu einer erheblichen Einschränkung der Alltagsfunktion und Lebensqualität der Patienten führen. Neben den beschriebenen kognitiven Defiziten leiden viele Patienten zusätzlich auch unter einer Fatigue-Symptomatik und Depressionen [36]. So überrascht es nicht, dass die erfolgreiche Therapie der HCV-Infektion zu einer Senkung der Mortalität, einer Verbesserung der Fatigue-Symptomatik und der Lebensqualität führen kann [36, 37]. Auch eine Verbesserung der Kognition nach erfolgreicher HCV-Therapie ist dokumentiert [35]. Im Zweifel sollten Patienten mit subjektiver kognitiver Verschlechterung bei HCV-Infektion deshalb niedrigschwellig auf kognitive Defizite gescreent und gegebenenfalls neuropsychologisch getestet werden. Eine HCV-Eradikation bietet bei dieser Patientengruppe die seltene Chance einer kausalen Therapie des kognitiven Defizits und sollte deshalb nicht verpasst werden.

\section{Myelitis}

Als Myelitis werden inflammatorische Veränderungen des Rückenmarks bezeichnet, die sowohl die weiße als auch die graue Substanz betreffen können. Man unterscheidet langstreckige inflammatorische Veränderungen von kurzstreckigen Formen der Myelitis (transverse Myelitis). Rezidivierende, transverse Myelitiden können im Kon- 
text einer HCV-Infektion auftreten. In einer Fallserie kam es bei HCV-positiven Patienten mit transverser Myelitis zu 2 bis 5 Rezidiven [38]. Andere chronisch-entzündliche ZNS-Erkrankungen - insbesondere die Neuromyelitis optica - sowie eine Kleingefäßvaskulitis wurden ausgeschlossen und stellen in dieser Konstellation wichtige Differenzialdiagnosen dar. Die Therapie erfolgt primär immunsuppressiv. Ob eine zusätzliche antivirale Therapie das Behandlungsergebnis dieser Patienten verbessert, ist aktuell unklar [39]. Bei der Pathogenese der Myelitis scheint aber eine HCVinduzierte Autoimmunität, wie auch bei anderen extrahepatischen Manifestationen, eine besondere Rolle $\mathrm{zu}$ spielen [40].

\section{Enzephalitis}

Verschiedene inflammatorische und demyelinisierende Erkrankungen des Hirnparenchyms sind - allesamt insgesamt selten - im Kontext der HCVInfektion beschrieben. Dabei handelt es sich um Fälle von Patienten mit akuter disseminierter Enzephalomyelitis (ADEM), mit Multiple-Sklerose-ähnlichen Läsionen sowie Enzephalitiden [41]. Die ADEM ist sowohl bei akuter als auch chronischer HCV-Infektion beschrieben [42]. In sehr seltenen Fällen ist das Auftreten fulminanter Enzephalitiden dokumentiert worden [43]. Ein mögliches therapeutisches Ansprechen auf antivirale Therapien ist bislang nicht beschrieben.

- HCV-assoziierte kognitive Defizite stellen eine häufige Komplikation dar und können einen relevanten Einfluss auf die Lebensqualität der Patienten haben.

- Kognitive Defizite bei der HCVInfektion lassen sich durch eine antivirale Therapie kausal therapieren.

- Myelitiden und demyelinisierende ZNS-Erkrankungen stellen sehr seltene Komplikationen der HCVInfektion dar.

\section{Die HCV-Infektion als Risikofaktor}

Die aktive HCV-Infektion erhöht das Risiko sowohl für den ischämischen als auch den hämorrhagischen Schlaganfall. Interessanterweise ist das Risiko, einen ischämischen Schlaganfall zu erleiden, auch unabhängig vom Vorliegen anderer kardiovaskulärer Risikofaktoren erhöht [44]. Besonders bei jüngeren Patienten erhöht eine HCV-Infektion das Risiko für intrazerebrale Blutungen bzw. hämorrhagische Schlaganfälle - möglichweise durch chronische vaskulitische Veränderungen der kleinen Gefäße [45]. Eine HCV-Eradikation senkt in dieser Patientengruppe das Risiko für ein erneutes Blutungsereignis [46]. Auch Patienten mit ischämischen Läsionen auf dem Boden einer HCV-assoziierten (in der Regel kryoglobulinämischen) ZNS-Vaskulitis profitieren von einer HCV-Eradikation, zusätzlich zu einer immunmodulierenden Therapie [47].

Ein interessanter Zusammenhang zur HCV-Infektion hat sich auch beim Morbus Parkinson gezeigt. Eine Infektion mit dem Hepatitis-B- oder dem HepatitisC-Virus erhöht das Risiko deutlich (RR 1,51-1,76), an einem Morbus Parkinson zu erkranken [48]. Ob dies auf eine direkte Neurotoxizität des HCV, zirkulierende proinflammatorische Zytokine oder andere Faktoren zurückzuführen ist, bleibt aktuell unklar. Allerdings konnten erste Arbeiten zeigen, dass eine erfolgreiche antivirale Therapie das Risiko, an einem Morbus Parkinson zu erkranken, zu senken vermag [49].

\section{Zusammenfassung}

Die HCV-Infektion ist eine im klinischen Alltag häufig anzutreffende Erkrankung, die auch aus neurologischer Sicht relevant ist. Mehr als jeder zweite HCV-infizierte Patient leidet unter neurologischen bzw. neuropsychiatrischen Folgen der Infektion. Dabei stellen Neuropathien und kognitive Defizite die häufigsten Komplikationen dar. Neuropathien können auch ohne Nachweis von Kryoglobulinen auftreten. Die HCV-Infektion sollte zukünftig in die Differenzialdiagnose von (Small-fiber-)Neuropathien und kogni- tiven Defiziten ebenso mit einbezogen werden wie in die Abklärung zerebrovaskulärer Ereignisse. Der Nachweis einer HCV-Infektion hat eine hohe therapeutische Konsequenz, denn die chronische Hepatitis C stellt heute in $>90 \%$ der Fälle eine kurativ behandelbare Erkrankung und damit einen behandelbaren Risikofaktor dar.

\section{Korrespondenzadresse}

\section{PD Dr. Katrin Hahn}

Klinik für Neurologie, Universitätsmedizin Charité

Charitéplatz 1, 10117 Berlin, Deutschland

katrin.hahn@charite.de

Funding. Open Access funding enabled and organized by Projekt DEAL.

\section{Einhaltung ethischer Richtlinien}

Interessenkonflikt. F. Kleefeld, G. Arendt, E. Neuen-Jacob, M. Maschke, I. Husstedt, M. Obermann, H. Schmidt und K. Hahn geben an, dass kein Interessenkonflikt besteht.

Für diesen Beitrag wurden von den Autoren keine Studien an Menschen oder Tieren durchgeführt. Für die aufgeführten Studien gelten die jeweils dort angegebenen ethischen Richtlinien.

Open Access. Dieser Artikel wird unter der Creative Commons Namensnennung 4.0 International Lizenz veröffentlicht, welche die Nutzung, Vervielfältigung, Bearbeitung, Verbreitung und Wiedergabe in jeglichem Medium und Format erlaubt, sofern Sie den/die ursprünglichen Autor(en) und die Quelle ordnungsgemäß nennen, einen Link zur Creative Commons Lizenz beifügen und angeben, ob Änderungen vorgenommen wurden.

Die in diesem Artikel enthaltenen Bilder und sonstiges Drittmaterial unterliegen ebenfalls der genannten Creative Commons Lizenz, sofern sich aus der Abbildungslegende nichts anderes ergibt. Sofern das betreffende Material nicht unter der genannten Creative Commons Lizenz steht und die betreffende Handlung nicht nach gesetzlichen Vorschriften erlaubt ist, ist für die oben aufgeführten Weiterverwendungen des Materials die Einwilligung des jeweiligen Rechteinhabers einzuholen.

Weitere Details zur Lizenz entnehmen Sie bitte der Lizenzinformation auf http://creativecommons.org/ licenses/by/4.0/deed.de.

\section{Literatur}

1. Petruzziello A et al (2016) Global epidemiology of hepatitis $C$ virus infection: an up-date of the distribution and circulation of hepatitis $C$ virus genotypes. WJG 22(34):7824-7840 
2. Sulkowski MS et al (2016) Effectiveness of simeprevir plus sofosbuvir, with or without ribavirin, in real-world patients with HCV genotype 1 infection. Gastroenterology 150(2):419-429

3. Zignego AL et al (1992) Infection of peripheral mononuclear blood cells by hepatitis $C$ virus. JHepatol 15(3):382-386

4. Ferri C et al (1993) Infection of peripheral blood mononuclear cells by hepatitis $C$ virus in mixed cryoglobulinemia. Blood 82(12):3701-3704

5. Ferri C et al (2007) HCV-related autoimmune and neoplastic disorders: the HCV syndrome. Dig Liver Dis 39(1):S13-21

6. Dammacco F et al (2000) The lymphoid system in hepatitis $C$ virus infection: autoimmunity, mixed cryoglobulinemia, and Overt B-cell malignancy. Semin Liver Dis 20(2):143-157

7. Monti G et al (1995) Cryoglobulinaemias: a multicentre study of the early clinical and laboratory manifestations of primary and secondary disease. GISC. Italian group for the study of cryoglobulinaemias. QJM 88(2):115-126

8. Brouet JC et al (1974) Biologic and clinical significance of cryoglobulins. A report of 86 cases. Am JMed 57(5):775-788

9. Gorevic PD et al (1980) Mixed cryoglobulinemia: clinical aspects and long-term follow-up of 40 patients. Am J Med 69(2):287-308

10. Nemni R et al (2003) Peripheral neuropathy in hepatitis $C$ virus infection with and without cryoglobulinaemia. J Neurol Neurosurg Psychiatry 74(9):1267-1271

11. Himoto T, Masaki T (2012) Extrahepatic manifestations and autoantibodies in patients with hepatitis Cvirusinfection. Clin Dev Immunol 2012:871401

12. Chiu WC et al (2014) Hepatitis C viral infection and the risk of dementia. Eur J Neurol 21(8):1068-e59

13. Cacoub P et al (2000) Extrahepatic manifestations associated with hepatitis $C$ virus infection. A prospective multicenter study of 321 patients. The GERMIVIC. Groupe d'etude et de recherche en medecine Interne et maladies infectieuses sur le virus de I'hepatite C. Medicine (Baltimore) 79(1):47-56

14. Lidove $O$ et al (2001) Hepatitis $C$ virus infection with peripheral neuropathy is not always associated with cryoglobulinaemia. Ann Rheum Dis 60(3):290-292

15. Zaltron $S$ et al (1998) High prevalence of peripheral neuropathy in hepatitis C virus infected patients with symptomatic and asymptomatic cryoglobulinaemia. Ital J Gastroenterol Hepato 30(4):391-395

16. Tembl Jl et al (1999) Neurologic complication associated with hepatitis $C$ virus infection. Neurology 53(4):861-864

17. Younis LK et al (2007) Immunohistochemical detection of HCV in nerves and muscles of patients with $\mathrm{HCV}$ associated peripheral neuropathy and myositis. Int J Health Sci 1(2):195-202

18. De Martino L et al (2003) Viral RNA in nerve tissues of patients with hepatitis Cinfection and peripheral neuropathy. Muscle Nerve 27(1):102-104

19. Russi $S$ et al (2018) HCV RNA genomic sequences and HCV-E2 glycoprotein in sural nerve biopsies from HCV-infected patients with peripheral neuropathy. Neuropathol Appl Neurobiol. https:// doi.org/10.1111/nan.12413

20. Biasiotta A et al (2014) Clinical, neurophysiological and skin biopsy findings in peripheral neuropathy associated with hepatitis $C$ virus-related cryoglobulinemia. J Neurol 261(4):725-731
21. Yoon MS et al (2011) Sensory neuropathy in patients with cryoglobulin negative hepatitis-C infection. J Neurol 258(1):80-88

22. Moretti R et al (2018) Hepatitis C-related cryoglobulinemic neuropathy: potential role of oxcarbazepine for pain control. BMC Gastroentero 18(1):19

23. Ballare $M$ et al (1995) A pilot study on the effectiveness of cyclosporine in type II mixed cryo globulinemia. Clin Exp Rheumatol 13(13):S201-3

24. Murai H et al (1995) Hepatitis C virus associated cryoglobulinemic neuropathy successfully treated with plasma exchange. ArtifOrgans 19(4):334-338

25. Lacaille F et al (1998) Hepatitis C associated with Guillain-Barre syndrome. Liver 18(1):49-51

26. Chin RL et al (2010) Demyelinating neuropathy in patients with hepatitis C virus infection. J Clin Neuromuscul Dis 11(4):209-212

27. Corcia P et al (2004) Improvement of a CIDP associated with hepatitis $C$ virus infection using antiviral therapy. Neurology 63(1):179-180

28. Tsuzaki $\mathrm{K}$ et al (2018) Remission of chronic inflammatory demyelinating polyneuropathy after hepatitis $C$ virus eradication with sofosbuvir and ledipasvir therapy. Muscle Nerve 58(5):E34-E36

29. Horsmans Y, Geubel AP (1995) Symptomatic myopathy in hepatitis-C infection without interferon therapy. Lancet 345(8959):1236-1236

30. Uruha A et al (2016) Hepatitis C virus infection in inclusion body myositis: a case-control study. Neurology 86(3):211-217

31. Takamiya $M$ et al (2019) Effect of intravenous immunoglobulin therapy on anti-NT5C1A antibody-positive inclusion body myositis after successful treatment of hepatitis C: a case report. eNeurologicalSci 16:100204

32. Burdette D et al (2012) Hepatitis C virus activates interleukin-1beta via caspase-1-inflammasome complex.J Gen Virol 93(2):235-246

33. Forton DM et al (2002) Hepatitis C and cognitive impairment in a cohort of patients with mild liver disease. Hepatology 35(2):433-439

34. McAndrews MP et al (2005) Prevalence and significance of neurocognitive dysfunction in hepatitis $C$ in the absence of correlated risk factors. Hepatology 41(4):801-808

35. Kleefeld F et al (2018) Interferon-free therapy in hepatitis $\mathrm{C}$ virus (HCV) monoinfected and HCV/HIV coinfected patients: effect on cognitive function, fatigue, and mental health. J Neuroviro 24(5):557-569. https://doi.org/10.1007/s13365018-0647-z

36. van der Meer AJ et al (2012) Association between sustained virological response and allcause mortality among patients with chronic hepatitis $C$ and advanced hepatic fibrosis. JAMA 308(24):2584-2593

37. Gerber Let al (2016) Effects of viral eradication with ledipasvir and sofosbuvir, with or without ribavirin, on measures of fatigue in patients with chronic hepatitis $C$ virus infection. Clin Gastroenterol Hepatol 14(1):156

38. Aktipi KM et al (2007) Severe recurrent myelitis in patients with hepatitis C virus infection. Neurology 68(6):468-469

39. Beuthien W, Mellinghoff HU, Kempis J (2005) Vasculitic complications of interferon-alpha treatment for chronic hepatitis $C$ virus infection: case report and review of the literature. Clin Rheumatol 24(5):507-515

40. Stubgen JP (2011) Immune-mediated myelitis associated with hepatitis virus infections. J Neuroimmunol 239(1-2):21-27
41. Bezerra MLE et al (2011) Hepatitis C virus: a rare manifestation-remitting relapsing central and peripheral demyelination. Neurol India 59(1):114-116

42. Sim JE et al (2012) A case of acute disseminated encephalomyelitisassociated with hepatitis C virus infection. Yonsei Med J 53(4):856-858

43. Seifert $F$ et al (2008) In vivo detection of hepatitis $\mathrm{C}$ virus (HCV) RNA in the brain in a case of encephalitis: evidence for HCV neuroinvasion. Eur J Neurol 15(3):214-218

44. Lee KKet al (2019) Global burden of atherosclerotic cardiovascular disease in people with hepatitis $C$ virus infection: a systematic review, meta-analysis, and modelling study. Lancet Gastroenterol Hepatol 4(10):794-804

45. Tseng CH et al (2015) Increased risk of intracerebral hemorrhage among patients with hepatitis C virus infection. Medicine 94(46):e2132

46. Arase $Y$ et al (2014) Impact of virus clearance for the development of hemorrhagic stroke in chronic hepatitis C. J Med Virol 86(1):169-175

47. SilvaFetal(2019) Newinsightsincryoglobulinemic vasculitis. J Autoimmun 105:102313

48. Pakpoor J et al (2017) Viral hepatitis and Parkinson disease: a national record-linkage study. Neurology 88(17):1630-1633

49. Su TH et al (2019) Antiviral therapy in patients with chronic hepatitis $\mathrm{C}$ is associated with a reduced risk of parkinsonism. Mov Disord 34(12):1882-1890 